Психология. Журнал Высшей школы экономики.

2016. T. 13. № 2. С. 410-420.

Короткие сообщения

\title{
ДВИЖЕНИЯ ГЛАЗ В ХОДЕ СПОНТАННОГО И КОНТРОЛИРУЕМОГО ОБНАРУЖЕНИЯ СЛОВ В БОЛЫШИХ БУКВЕННЫХ МАССИВАХ
}

\author{
С.А. ЯЗЫКОВ ${ }^{\mathrm{a}}$, М.В. ФАЛИКМАН ${ }^{\mathrm{ab}, \mathrm{c}}$ \\ а Московский государственный университет им. М.В. Ломоносова, 119991, Россия, Москва, \\ Ленинские горы, Ә. 1.

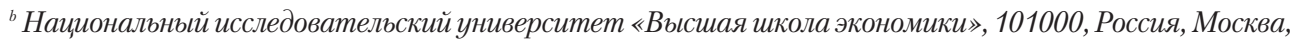 \\ ул. Мясницкая, Ә. 20 \\ ' Институт общественных наук РАНХиГС, 119571, Россия, Москва, пр. Вернадского, д. 82, стр. 1.
}

\begin{abstract}
Резюме
Целью работы было изучение возможностей и ограничений нисходящих влияний на обработку зрительных стимулов на материале задачи зрительного поиска букв и слов в случайных рядах букв. В наших недавних экспериментах было показано, что присутствие слов в буквенных массивах, в которых наблюдатель решает задачу поиска букв, не влияет на продуктивность решения этой задачи вне зависимости от того, замечает ли испытуемый слова. Мы установили, что спонтанное выделение слов и их произвольное отыскание в соответствии с инструкцией задействуют разные стратегии поиска, меняющие характер движений глаз. Спонтанное обнаружение слов при поиске букв не имеет значимых глазодвигательных коррелятов, в то время как контролируемый поиск слов увеличивает число фиксаций как в пределах слов, так и между ними. В дополнительном исследовании мы показали, что знание категориальной принадлежности слов не помогает испытуемым в выполнении задания на поиск слов; по всей видимости, в этом случае сначала происходит установление границ и выделение слов в стимульном материале и только затем процесс их категоризации. Таким образом, к обнаружению слов в буквенных массивах могут приводить, по крайней мере, два различных процесса: спонтанного обнаружения слова, опосредованный кодированием отдельных букв, и контролируемого выделения слова, опосредованный обработкой информации о слове как о целостной перцептивной единице.
\end{abstract}

Ключевые слова: зрительный поиск, перцептивные стратегии, перцептивная задача, регистрация движений глаз, эффект превосходства слова, тест Мюнстерберга. 
В современной когнитивной психологии широко обсуждается вопрос о возможностях и ограничениях так называемых нисходящих влияний на обработку зрительной информации. Источником этих влияний может выступать как прошлый опыт человека, так и цели и стратегии решения им перцептивных задач (Печенкова, Фаликман, 2010). Разграничение этих источников не всегда оказывается возможным на основе результатов экспериментов с традиционными поведенческими мерами, такими, как продуктивность решения задачи и время ответа, и требует использования дополнительных объективных показателей перцептивной активности.

В основу данного исследования положен «эффект превосходства слова» - один из устойчивых психологических эффектов, впервые описанный Дж.М. Кеттеллом в конце XIX в. (Cattell, 1886), а в XX в. сопровождавший развитие когнитивной психологии с ее первых шагов, начиная с работы Дж. Рейхера (Reicher, 1969) и опубликованной в первом выпуске журнала «Cognitive Psychology» статьи Д. Уилера (Wheeler, 1970). «Превосходство» слова заключается в том, что буквы, предъявленные в составе слов, опознаются точнее и быстрее в сравнении со случайными наборами букв и изолированно предъявленными буквами.

Существование подобного эффекта указывает на то, что зрительное восприятие организовано как многоуровневая система. Ключевой особенностью уровневого описания перцепции является предположение о возможности нисходящих влияний со стороны сложных, высокоуровне- вых форм переработки информации на протекание процесса восприятия на нижележащих уровнях.

Часто взаимодействие между уровнями представляется в качестве автоматического. Известный пример такого взгляда на организацию межуровневых связей - нейросетевая модель интерактивной активации (Interactive Activation Model) Дж. Макклелланда и Д. Румельхарта (McClelland, Rumelhart, 1981). Она включает три уровня, задействованных в зрительном опознании слова: 1) простых признаков; 2) букв и 3) слов, между которыми существуют двунаправленные связи, такие, что активация узла слова способствует активации входящих в это слово букв и формирующих его признаков и тормозит активацию остальных, не связанных с ним единиц. При этом первоначальную активацию слова обеспечивают исключительно простые зрительные признаки на перцептивном входе. Другие авторы предполагают, что выбор единиц, организующих восприятие, происходит произвольно: например, В. Вундт (2007) считал эффект превосходства слова следствием работы механизма внимания - апперцепции, а Ю.Б. Гиппенрейтер (1983) предположила, что ведущий уровень организации перцептивного процесса и структура перцептивных единиц определяются целью перцептивного действия, т.е. стоящей перед человеком задачей. Точку зрения Ю.Б. Гиппенрейтер поддерживают результаты исследований М.В. Фаликман (2001) и В.Ю. Степанова (2011), в которых возникновение эффекта превосходства слова в условиях быстрого последовательного предъявления 
зрительных стимулов требовало предварительного знания о том, что стимульный материал будет организован в слова.

Чтобы проверить предположение о возможности эффективного укрупнения перцептивных единиц в более естественных для человека условиях одновременного предъявления всех букв слова, мы провели исследование на модифицированном стимульном бланке теста Бурдона, добавив в него слова (Фаликман и др., 2013; Falikman, 2014). Испытуемые в этом исследовании получали задание на поиск заранее заданной буквы среди не разделенных пробелами букв в одном из трех типов бланков, представлявших собой буквенные массивы, организованные в 10 рядов по 60 символов. В бланках первого типа ряды букв содержали слова, и при этом целевая буква всегда находилась в словах (ЭГ1). Бланки второго типа также содержали слова, но целевая буква всегда располагалась вне слов (ЭГ2). Бланки третьего типа слов не содержали (КГ). О наличии слов испытуемые не были осведомлены. Значимых различий между тремя группами испытуемых по продуктивности выполнения задачи обнаружено не было, однако если $65 \%$ испытуемых из ЭГ1 замечали слова, то в ЭГ2 заметили слова $37.5 \%$ испытуемых. При разделении групп по критерию обнаружения слов значимые различия в продуктивности также отсутствовали. В следующем исследовании на том же стимульном материале мы предупреждали испытуемых о наличии слов и их расположении относительно целевых букв, но это никак не сказалось на полученных результатах: они не отличались от результатов первого эксперимента, следовательно, испытуемые не использовали эти сведения для решения поставленной задачи.

Мы интерпретировали эти результаты как диссоциацию нисходящих процессов и нисходящих влияний на решение перцептивной задачи (Falikman, 2014): испытуемые замечают слова (что требует обращения к опыту использования этих слов), но слова не оказывают влияния на скорость нахождения целевых букв. Эти результаты расходятся с традиционными представлениями об эффекте превосходства слова. Если повышение эффективности обработки информации о букве в составе слова является «побочным продуктом» обработки информации о слове, то наличие спрятанных в буквенных массивах слов ускорило бы выполнение задания. В то же время обнаруженные слова не привлекают к себе внимания (не навязывают неэффективного укрупнения перцептивной единицы), иначе следовало бы ожидать снижения продуктивности поиска целевой буквы.

В настоящем исследовании мы добавили к описанным экспериментальным условиям дополнительное условие с заданием на поиск слов, а также новую зависимую переменную - параметры движений глаз. Мы рассчитывали выявить глазодвигательные корреляты спонтанного обнаружения слов в буквенных массивах (предположив, что наличие слов одновременно помогает и осложняет поиск целевой буквы и что эффекты аннулируют друг друга), а также проверить, отличается ли заведомо контролируемое 
обнаружение слов в буквенных массивах в соответствии с поставленной задачей (при задании на поиск слов - так называемом тесте Мюнстерберга) от автоматического обнаружения слов при решении задачи поиска букв. Таким образом, мы ожидали увеличения количества фиксаций на областях слов при задании на поиск букв в сравнении с областями целевых букв, не являющимися в то же время областями слов, а также «перегонами» между целевыми буквами. Кроме того, предполагалось, что будут выявлены различия в количестве фиксаций при выполнении заданий на поиск слов и поиск букв.

\section{Методика}

Стимульный материал. В исследовании использовались стимульные бланки, идентичные бланкам, применявшимся в наших предыдущих работах (Фаликман и др., 2013; Falikman, 2014). Они создавались с помощью специальной компьютерной программы-генератора с использованием заранее подготовленных «словарей» стимулов и содержали 10 строк по 60 строчных букв русского алфавита без пробелов. В двух бланках в эти строки были включены 24 шестибуквенных слова русского языка из среднего диапазона частотности, при этом общее количество букв и строк оставалось одним и тем же.

Всего мы использовали три типа бланков в четырех условиях, в каждом из которых участвовала отдельная группа испытуемых: 1) выполнение задания на поиск буквы Т в буквенных массивах со словами, где целевая буква всегда входила в состав слов (ЭГ1); 2) выполнение задания на поиск буквы Т в буквенных массивах со словами, где целевая буква никогда не входила в состав слов (ЭГ2); 3) выполнение задания на поиск буквы Т в буквенных массивах, не содержавших слова (ЭГЗ); 4) выполнение задания на поиск слов в буквенных массивах, в которых каждое из целевых слов содержит букву Т (ЭГ4). Каждому испытуемому предъявлялся единственный бланк в соответствии с условием.

Стимулы предъявлялись на мониторе компьютера и были напечатаны шрифтом Times New Roman, кегль 27. Угловые размеры всего текста составляли $30^{\circ} \times 20^{\circ}$ по горизонтали и вертикали соответственно. Каждая строчная буква имела высоту 6 мм, что приблизительно соответствовало 0.55 угловым градусам для глаза испытуемого.

Испытуемые. В исследовании приняли участие 32 человека, студенты и аспиранты МГУ, в возрасте 19-25 лет (средний возраст - 21 год) с нормальным или скорректированным до нормального зрением, все правши. В каждую экспериментальную группу вошло 8 испытуемых.

Аппаратура. Исследование проводилось на установке для регистрации движений глаз «iView X system» фирмы SMI.

Процедура. Испытуемые проходили исследование в индивидуальном порядке. Эксперимент начинался с процедуры калибровки, занимавшей от одной до десяти минут. Затем устно проговаривалась экспериментальная инструкция. Каждому испытуемому давалось только одно 
задание, либо на поиск слов, либо на поиск букв. Стимульный материал предъявлялся на экране в течение одной минуты. О нахождении новой целевой буквы или слова испытуемый отчитывался нажатием на клавишу «Enter». По завершении работы испытуемым ЭГ1, ЭГ2 и ЭГЗ предлагалось ответить на вопрос с вынужденным выбором («да»/ «нет»): заметили ли они в буквенных строках слова, а в случае положительного ответа предлагалось припомнить слова, которые они заметили.

Обработка результатов. На стимульном материале выделялись зоны интереса (Area of Interest, AOI). Всего было выделено 72 AOI, 24 из которых соответствовали целевым объектам, 24 - расположению слов в предъявляемых на экране буквенных строках, 24 - областям «перегонов» между словами. Для ЭГ1 и ЭГ4 области целевого объекта совпадали с областями слов, так что на них выделялось по 48 AOI. Для ЭГЗ так же было выделено только 48 АОІ, так как в бланках этой группы не было слов. Таким образом, в совокупности, по данным всех испытуемых, было выделено 1728 зон интереса (48 AOI * 24 испытуемых +72 AOI * 8 испытуемых). Площади всех AOI были одинаковы и содержали по 13104 пикселя.

Для каждой AOI считалось общее число фиксаций испытуемого на ней. Из анализа данных были исключены необследованные АOI - те области, на которые не приходилось ни одной фиксации. Также были исключены AOI, количество фиксаций на которых превышало 3 стандартных отклонения.

По условиям ЭГ1 и ЭГ4 анализировалась продуктивность выполне- ния экспериментальных заданий (по числу нажатий на клавишу «Enter»). Статистическая обработка результатов производилась с помощью программы SPSS Statistics 20.

\section{Результаты}

Продуктивность. Были обнаружены значимые различия по критерию продуктивности (сравнение ЭГ1 и ЭГ4, критерий Стьюдента для независимых выборок $t=4.025, p<0.01$, двусторонний): задание на поиск букв выполнялось эффективнее (среднее число найденных целевых стимулов - 18, или 75\% всех целевых стимулов), чем задание на поиск слов (среднее число найденных целевых стимулов - 10, или $46 \%$ всех целевых стимулов) на одном и том же стимульном материале. Применение параметрического критерия обусловлено тем, что распределение результатов в обеих выборках значимо не отличалось от нормального (для ЭГ1: $z=0.345, p=1.000$; для ЭГ2: $z=0.464, p=0.982)$, а дисперсии выборок значимо не различались (тест Левина на равенство дисперсий: $F=0.523, p=0.482)$.

Для статистической обработки данных по количеству фиксаций использовался непараметрический критерий Манна-Уитни, поскольку распределение данных отличалось от нормального.

Количество фиксащий. Был обнаружен ряд значимых различий в количестве фиксаций между исследуемыми группами: области слов при задании на поиск слов фиксируются значимо больше, чем любые другие области при выполнении задания на поиск букв, а также чем 
области «перегонов» между словами при выполнении задания на поиск слов. При выполнении задания на поиск букв на область целевого объекта так же приходится больше фиксаций, чем на область перегонов этого же условия, при этом количество фиксаций на областях целевых объектов и перегонов между условиями поиска букв не различаются. Данные обо всех различиях в количестве фиксаций на разных областях с указанием уровня значимости различий представлены в таблице 1.

Из представленных в таблице 1 значений для дальнейшей интерпре- тации в соответствии с выдвинутыми гипотезами нам важны следующие сравнения: $1-2,1-8,2-9,8-9$, что позволит дать ответ на вопрос, различается ли автоматическое и контролируемое обнаружение слов, а также 3-4, 2-7, которые помогут выяснить, существуют ли глазодвигательные корреляты автоматического обнаружения слов и вмешиваются ли слова в процесс обнаружения букв при выполнении задания на поиск букв. После применения поправки Бонферрони для множественных сравнений уровень значимости $-0.05 / 6=0.008$.

Таблица 1

Уровни значимости различий между всеми экспериментальными подгруппами (критерий Манна-Уитни, двухсторонние квантили)

\begin{tabular}{|c|c|c|c|c|c|c|c|c|c|c|}
\hline & \multicolumn{2}{|c|}{$\begin{array}{c}\text { Поиск букв, } \\
\text { целевая буква } \\
\text { в словах, ЭГ1 }\end{array}$} & \multicolumn{3}{|c|}{$\begin{array}{l}\text { Поиск букв, целевая } \\
\text { буква вне слов, ЭГ2 }\end{array}$} & \multicolumn{2}{|c|}{$\begin{array}{c}\text { Поиск букв, без } \\
\text { слов, ЭГЗ }\end{array}$} & \multicolumn{2}{|c|}{ Поиск слов, ЭГ4 } \\
\hline & & $\begin{array}{c}1 \\
\text { перегоны }\end{array}$ & $\begin{array}{c}2 \\
\text { цель }\end{array}$ & $\begin{array}{c}3 \\
\text { слова }\end{array}$ & $\begin{array}{c}4 \\
\text { перегоны }\end{array}$ & $\begin{array}{c}5 \\
\text { цель }\end{array}$ & $\begin{array}{c}6 \\
\text { перегоны }\end{array}$ & $\begin{array}{c}7 \\
\text { цель }\end{array}$ & $\begin{array}{c}8 \\
\text { перегоны }\end{array}$ & $\begin{array}{c}9 \\
\text { цель }\end{array}$ \\
\hline & $\begin{array}{c}\text { Среднее } \\
\text { количество } \\
\text { фиксаций }\end{array}$ & 2.78 & 3.16 & 2.95 & 2.72 & 3.20 & 2.58 & 3.16 & 3.36 & 3.83 \\
\hline 1 & 2.78 & - & 0.015 & 0.208 & 0.566 & 0.003 & 0.363 & 0.012 & 0.005 & 0.001 \\
\hline 2 & 3.16 & & - & 0.220 & 0.003 & 0.522 & 0.001 & 0.878 & 0.507 & 0.002 \\
\hline 3 & 2.95 & & & - & 0.064 & 0.068 & 0.022 & 0.217 & 0.087 & 0.001 \\
\hline 4 & 2.72 & & & & - & 0.001 & 0.864 & 0.003 & 0.001 & 0.001 \\
\hline 5 & 3.20 & & & & & - & 0.001 & 0.663 & 0.898 & 0.007 \\
\hline 6 & 2.58 & & & & & & - & 0.001 & 0.001 & 0.001 \\
\hline 7 & 3.16 & & & & & & & - & 0.536 & 0.002 \\
\hline 8 & 3.36 & & & & & & & & - & 0.028 \\
\hline 9 & 3.83 & & & & & & & & & - \\
\hline
\end{tabular}

Примечание. В строках и столбцах таблицы обозначены одни и те же экспериментальные условия, в клетках таблицы даны результаты сравнения между ними. 
Субъективные отчетьл. 88\% испытуемых отчитались о том, что заметили слова в ЭГ1, 13\% испытуемых отчитались о том, что заметили слова в ЭГ2, и никто не отчитался о том, что заметил слова в ЭГЗ (где стимульный материал не содержал слов). Таким образом, число испытуемых, заметивших слова в ЭГ1, значимо отличалось от числа испытуемых, заметивших слова в ЭГ2 (критерий Хи-квадрат Пирсона = $9.000, p<0.01$, двусторонний) и ЭГЗ (критерий Хи-квадрат Пирсона = 12.444, $p<0.001$, двусторонний). Число испытуемых, заметивших слова в ЭГ2 и ЭГЗ, значимо не различалось (критерий Хи-квадрат Пирсона $=1.067, p=0.302$, двусторонний).

\section{Обсуждение}

Первый из полученных нами важных результатов - различие в продуктивности отыскания букв и слов при выполнении соответствующих заданий на одном и том же стимульном материале, несмотря на то, что почти все испытуемые, выполнявшие задание на поиск букв, заметили слова. Различие становится еще более ощутимым, если учесть, что слова охватывают в шесть раз больше стимульного материала (целевые буквы - 4\% символов, среди которых осуществляется поиск; целевые слова - 24\% символов). Это свидетельствует о том, что зрительный поиск при выполнении этих заданий осуществлялся по-разному. Кроме того, обнаружились различия в характере фиксаций при решении задачи поиска слов по сравнению с задачей поиска букв. Количество фиксаций на словах и на «перегонах» между словами значимо выше для условия поиска слов по сравнению с условием поиска букв на том же стимульном материале. По всей видимости, низкая продуктивность при выполнении задания на поиск слов связана с избираемой стратегией зрительного поиска (последовательный побуквенный поиск границ слова), а не с необходимостью перцептивного выделения и обработки слов (количество фиксаций на словах и на «перегонах» в рамках одного условия значимо не различаются). Сравнение всех трех условий поиска букв показывает, что они сходны друг с другом по характеру фиксаций. Количество фиксаций на областях слов, не являющихся в то же время областями целевого объекта, значимо не отличается от количества фиксаций на «перегонах» между целевыми буквами, а количество фиксаций на целевых буквах, включенных в слова, не отличается от количества фиксаций на целевых буквах в стимульном материале, где слова отсутствовали. Таким образом, обнаружение слов в описанных условиях не требует дополнительных когнитивных затрат, которые сказались бы на увеличении количества фиксаций. Мы предполагаем, что обнаружение слов при выполнении задания на поиск букв в буквенных массивах происходит не просто автоматически, но спонтанно: слово в этом случае активируется как образ памяти, не включенный в актуальный процесс восприятия в качестве средства и не вмешивающейся в него. Вместе с тем при поиске целевых букв в массивах, содержащих слова на «перегонах» между целевыми буквами, эти 
слова фиксируются чаще случайных последовательностей букв на уровне тенденции при $p<0.06$ (но не чаще и не реже, чем слова при поиске букв, входящих в состав слов). Это заставляет допустить, что выделенные, но не замеченные слова могут кодироваться в ходе поиска.

Подобную интерпретацию можно было бы распространить и на выполнение задания на поиск слов: сначала слово соотносится со схемой памяти вне привязки к перцептивному опыту, например, как одно из следствий фонологического кодирования отдельных букв, затем, для уже активированной схемы слова, эксплицируются зрительные перцептивные гипотезы и поиск производится в соответствии с ними. Увеличение количества фиксаций на перегонах между словами можно объяснить тем, что у испытуемых нет информации о точном местоположении слова в стимульном материале и о его границах и они могут только примерно выделить его, опираясь на время активации схемы в памяти, а также на неверно активированные схемы слов. Но в дополнительном эксперименте $^{1}$ с использованием бланковой методики без регистрации движений глаз было показано, что решению задачи поиска слов не способствует преднастройка «сверху», а именно указание категории слов, которые необходимо отыскать в буквенных рядах. Поскольку установить принадлежность слова к категории можно, только выделив это слово, предактивация слова в «ментальном лексиконе» не ускоряет процесса установления его границ.

Мы предполагаем, что контролируемое выделение слов происходит иначе, чем их спонтанное обнаружение: оно опосредовано стратегией решения задачи и связанной с ней организацией процесса восприятия. Полученные нами данные косвенно указывают на то, что опознание слова осуществляется на нескольких уровнях, участие этих уровней в обработке информации о слове модулируется постановкой задачи. При определенных условиях - при «спонтанном обнаружении слова»эффект превосходства слова не наблюдается за счет того, что уровни построения перцептивного образа, связанные с его возникновением, оказываются исключены из процесса обнаружения слова.

\section{Выводы}

1. К обнаружению слов в случайных буквенных массивах могут приводить, по крайней мере, два различных процесса: спонтанного обнаружения слова, опосредованный кодированием отдельных букв, и

\footnotetext{
${ }^{1}$ В данном эксперименте, выполненном в рамках дипломного исследования С.А. Языкова, 15 испытуемых (средний возраст - 19 лет) решали задачу поиска среднечастотных слов русского языка в буквенных массивах, аналогичных использованным в основной серии экспериментов. При этом все слова принадлежали к одной категории («одежда»). Значимых отличий от решения задачи поиска слов, не относящихся к одной категории, обнаружено не было: $\mathrm{F}(1,40)=2.634, p=$ 0.112, хотя все испытуемые отчитались, что знание о категориальной принадлежности слов помогало им решать задачу (Языков, 2015).
} 
контролируемого выделения слова, опосредованный обработкой информации о слове как о целостной перцептивной единице.

2. Задание на поиск слов приводит к изменению стратегии зрительного поиска в сравнении с заданием на поиск букв и сказывается на равномерном увеличении числа фиксаций на всем стимульном материале.

3. Спонтанное обнаружение слов в буквенных массивах при решении задачи поиска букв не вызывает значимого увеличения количества фиксаций на словах, содержащихся в буквенных рядах.

4. Особенности движений глаз при поиске букв и слов в одних и тех же буквенных массивах позволяют предположить, что выделение слова обусловлено не специальной «операцией внимания» как укрупнения единиц обработки информации, а стратегией поиска.

\section{Литература}

Вундт, В. (2007). Введение в психологию. М.: КомКнига.

Гиппенрейтер, Ю. Б. (1983). Деятельность и внимание. В кн. А. В. Запорожец (ред.), А.Н. Леонтьев и современная психология (с. 165-177). М.: Изд-во Московского университета.

Печенкова, Е. В., Фаликман, М. В. (2010). Решение перцептивной задачи как взаимодействие между восходящими и нисходящими процессами переработки зрительной информации. Теоретическая и экспериментальная психология, 3(3), 52-65.

Степанов, В. Ю. (2011). Структурные единицы внимания в условиях быстрой смены зрительных стимулов (Кандидатская диссертация, Московский государственный университет имени М.В. Ломоносова, Москва).

Фаликман, М. В. (2001). Динамика внимания в условиях быстрого последовательного предъявления зрительных стимулов (Кандидатская диссертация, Московский государственный университет им. М.В. Ломоносова, Москва).

Фаликман, М. В., Поминова, А. М., Языков, С. А. (2013). Буквы в словах и слова в буквах: к вопросу о перцептивных единицах. В кн. Е. В. Печенкова, М. В. Фаликман (ред.), Когнитивная наука в Москве: новые исследования (с. 298-303). М.: БукиВеди.

Языков, С. А. (2015). Внимание в задаче зрительного поиска в буквенных массивах (Дипломная работа, Московский государственный университет имени М.В. Ломоносова, Москва).

Cattell, J. M. (1886). The time it takes to see and name objects. Mind, 11, 63-65.

Falikman, М. (2014). Attention and chunking in visual search among letter stimuli. Психология. Журнал Высшей школы экономики, 11(2), 150-159.

McClelland, J., \& Rumelhart, D. (1981). An interactive activation model of context effects in letter perception: Part 1. An account of basic findings. Psychological Review, 88(5), 375-407.

Reicher, G. M. (1969). Perceptual recognition as a function of meaningfulness of stimulus material. Journal of Experimental Psychology, 81(2), 275-280.

Wheeler, D. D. (1970). Processes in word recognition. Cognitive Psychology, 1(1), 59-85.

Языков Сергей Александрович - выпускник, факультет психологии, МГУ имени М.В. Ломоносова.

Контакты: yazykov.sa@gmail.com 
Фаликман Мария Вячеславовна - старший научный сотрудник, кафедра теоретической и прикладной лингвистики, филологический факультет, МГУ имени М.В. Ломоносова; ведущий научный сотрудник, лаборатория когнитивных исследований, Национальный исследовательский университет «Высшая школа экономики»; старший научный сотрудник, лаборатория когнитивных исследований, Институт общественных наук РАНХиГС; кандидат психологических наук.

Контакты: maria.falikman@gmail.com

\title{
Eye Movements in Spontaneous and Controlled Word Detection in Letter Arrays
}

\author{
Sergey A. Yazykov ${ }^{\mathrm{a}}$, Maria V. Falikman ${ }^{\mathrm{ab}, \mathrm{c}}$ \\ ${ }^{a}$ Lomonosov Moscow State University, 1 Leninskie Gory, Moscow, 119991, Russian Federation \\ ${ }^{b}$ National Research University Higher School of Economics, 20 Myasnitskaya str., Moscow, 101000, \\ Russian Federation \\ ${ }^{c}$ Institute for Humanities, Russian Academy of National Economy and Public Administration, 82/1, \\ Prospect Vernadskogo, Moscow, 119571, Russian Federation
}

\begin{abstract}
The aim of this study was to analyze the scope and limitations of top-down influences upon visual information processing (during visual search for letters and words in random letter arrays). Previously we have demonstrated that the presence of words in large letter arrays does not affect the efficiency of visual search for a target letter in these arrays. The current study shows that spontaneous word extraction during visual search for target letters and controlled word extraction during visual search for words are mediated by different perceptual strategies. Spontaneous word detection is not accompanied by specific eye movements, whereas the words search task produces an increase of the number of eye fixations over all the letter array, both within and between words. In an additional study we demonstrate that categorical set does not affect the efficiency of visual search for words in random letter arrays: it seems that observers first extract words from letter arrays and then categorize detected words. Thus, there appears to exist two distinct mechanisms providing for the detection of words in letter arrays. The first mechanism is based on the processing of separate letters, whereas the second one is based on the processing of words as perceptual chunks.
\end{abstract}

Keywords: visual search, perceptual strategies, perceptual task, eye movements, word superiority effect, Muensterberg's test.

\section{References}

Cattell, J. M. (1886). The time it takes to see and name objects. Mind, 11, 63-65.

Falikman, M.V. (2014). Attention and chunking in visual search among letter stimuli. Psychology. Journal of the Higher School of Economics, 11(2), 150-159. 
Falikman, M. V. (2001). Dinamika vnimaniya v usloviyakh bystrogo posledovatel'nogo pred"yavleniya zritel'nykh stimulov [Dynamics of attention in rapid serial visual presentation] (Ph.D. dissertation, Lomonosov Moscow State University, Moscow).

Falikman, M. V., Pominova, A. M., \& Yazykov, S. A. (2013). Bukvy v slovakh i slova v bukvakh: K voprosu o pertseptivnykh edinitsakh [The letters in the words and the words in the letters]. In E. V. Pechenkova \& M. V. Falikman (Eds.), Kognitivnaya nauka v Moskve: Novye issledovaniya [Cognitive science in Moscow: New research] (pp. 298-303). Moscow: BukiVedi.

Gippenreiter, Yu. B. (1983). Deyatel'nost' i vnimanie [Activity and attention]. In A. V. Zaporozhets (Ed.), A.N. Leontiev i sovremennaya psikhologiya [Leontiev and contemporary psychology] (pp. 165-177). Moscow: Moscow University Press.

McClelland, J., \& Rumelhart, D. (1981). An interactive activation model of context effects in letter perception: Part 1. An account of basic findings. Psychological Review, 88(5), 375-407.

Pechenkova, E. V., \& Falikman, M. V. (2010). Perceptual task decision as an interaction between ascending and descending mechanisms for visual information processing. Teoreticheskaya $i$ Eksperimentalnaya Psikhologiya, 3(3), 52-65.

Reicher, G. M. (1969). Perceptual recognition as a function of meaningfulness of stimulus material. Journal of Experimental Psychology, 81(2), 275-280.

Stepanov, V. Yu. (2011). Strukturnye edinitsy vnimaniya v usloviyakh bystroi smeny zritel'nykh stimulov [Structural effects of attention in rapid serial visual presentation] (Ph.D. dissertation, Lomonosov Moscow State University, Moscow).

Wheeler, D. D. (1970). Processes in word recognition. Cognitive Psychology, 1(1), 59-85.

Wundt, W. (2007). Vvedenie v psikhologiyu [Introduction to psychology]. Moscow: KomKniga. (transl. of: Wundt, W. (1911). Einführung in die Psychologie. Leipzig: Dürr (in German)).

Yazykov, S. A. (2015). Vnimanie v zadache zritel'nogo poiska v bukvennykh massivakh [Attention in visual search tasks within large letter arrays] (Thesis, Lomonosov Moscow State University, Moscow).

Sergey A. Yazykov - Faculty of Psychology, Lomonosov Moscow State University. E-mail: yazykov.sa@gmail.com

Maria V. Falikman - senior research fellow, Faculty of Philology, Lomonosov Moscow State University; leading research fellow, Cognitive Research Lab, National Research University Higher School of Economics; senior research fellow, Institute for Humanities, Russian Academy of National Economy and Public Administration, Ph.D.

E-mail: maria.falikman@gmail.com 